\title{
Increasing Discovery in Research, Design, and Other Processes with Artificial General Intelligence and General Collective Intelligence
}

Andy E. Williams

Nobeah Foundation, Nairobi, Kenya

\begin{abstract}
Any system with repeatable behavior can potentially be defined with the minimal set of functions that might be composed to represent the entirety of that behavior. The states accessible through these functions then forms a "functional state space" through which the system moves. Since functional states spaces can be used to represent every problem domain from physics, to communications, to business operations, to the human cognition itself, a general approach to not only research but design and all other processes of discovery that is applicable to all domains can potentially be defined to radically increase capacity for discovery in each domain.
\end{abstract}

\section{Keywords}

Artificial General Intelligence, General Collective Intelligence, research impact, innovation

\section{Introduction}

Any system with repeatable behavior can potentially be defined with the minimal set of functions that might be composed to represent the entirety of that behavior. The states accessible through these functions then forms a "functional state space" through which the system moves. Solving any problem with such a system involves finding some path through functional state space that is required to change from an initial state to a target state. The Human-Centric Modeling Framework (HCFM) [1] defines a methodology for applying this approach to any system.

The Functional Modeling Framework (FMF) [2] developed using this modeling approach defines a functional model for cognition, in which the "conceptual space" is the functional state space within which the cognitive system navigate. In this functional model the level of general problem-solving ability of a system of cognition is given by the volume of conceptual space that can be navigated per unit time. All other functional domains exist within that conceptual space, and the problem-solver's level of general problem-solving ability in the domain of that functional state space is determined by the volume of that functional state space the problem-solver can navigated per unit time.

In this way, all research, all design of new systems, all systems by which development, or manufacturing, or by which any other system of change impacting processes, and all other aspects of discovery for such systems then becomes a matter of defining a process by which the regions of state space that are accessible can be expanded, the resolution at which points in functional state space can be located can be increased, and the rate at which paths through the functional state space can be navigated can be increased. Just as arithmetic operations radically increase ability to navigate the entire space of real numbers, as opposed to memorizing sets of numbers and their relationships, defining the set of operations in functional state space that span a given domain can radically increase the ability of an individual's cognition to navigate that domain as opposed to memorizing intellectual relationships within it. Since functional states spaces can be used to represent every problem domain from physics, to communications, to business operations, to the human cognition itself, a general approach to not only research but design and all other processes of discovery that is applicable to all domains can potentially be defined to radically increase capacity for discovery in each domain.

\section{Related Work}


General Collective Intelligence or GCI has been described as a platform that organizes individual humans into a single collective intelligence with the potential capacity for exponentially greater general problem-solving ability than that of any individual in the group [3]. Though others have described the concept of a general collective intelligence factor (c factor) [4], though at least one other has defined a model for a general collective intelligence factor [5], and though others have described the concept of a collective super intelligence [6], to the author's knowledge the author's own work is the only model that defines the specific mechanisms required for a GCI to have the capacity to achieve super intelligence in that general collective intelligence factor. Because of this, and because this model is so new, all of the existing literature surrounding GCI refers to the author's own published work, or preprints of the author's unpublished work that are still under review.

\section{Representing Discovery in Functional State Space}

In functional state space discovery is represented by new reasoning processes that lead to concepts outside of currently accessible conceptual space, and it is represented by an increase in number of reasoning processes defining relationships between concepts so that the resolution at which points in conceptual space can be located is increased [3].

All functional state spaces provide mechanisms for defining the boundaries of any given set of regions in that space. And all functional state spaces provide mechanisms for defining the "physics" through which the functions of the system interact with those regions. Determining whether new reasoning processes represent truth can be achieved through comparing simulated behavior of a given region according to all the "physics" represented by those reasoning processes, and comparisons with patterns of actual outcomes for similar regions to detect patterns of behavior within that functional state space that are "unphysical" [9]. Such pattern detection might be performed at orders of magnitude greater speed and scale on behalf of an individual by an AGI based intelligent agent that combines all AI solutions into a single library that it might use to increase its general problem-solving ability [10]. In the case of a group, such pattern detection might be performed across all experiments in all domains at even greater speed and scale by employing a GCI to orchestrate cooperation between an unlimited number of intelligent agents. That GCI might also combine all CI solutions into a single library that it might use to increase its general problem-solving ability as well [11].

\section{Defining Capacity for Discovery in Functional State Space}

This functional model suggests that capacity for discovery in conceptual space is defined by the volume of conceptual space that can be navigated per unit time [3]. Since all functional state spaces are sub-spaces of conceptual space, the volume of conceptual space that can be navigated per unit time within the sub-space defined by the functional state space of a given domain, is predicted by this model to be the capacity for discovery within that domain.

\section{Increasing Capacity for Discovery in Functional State Space}

This functional model suggests that the rate at which problem-solving can occur in the conceptual space of the individual, or in the collective conceptual space of the collective cognition, is determined by the system's capacity to navigate complexity, the number of reasoning paths the system has the capacity to navigate, as well as the rate at which value can be created and the volume of value that can be created in order to sustain that navigation process [3].

Creating functional state spaces for each domain creates the potential to incorporate artificial intelligence (AI) into all research and design processes to serve the role of a vastly more powerful "artificial intuition". Human beings can intuitively detect representations of still or moving physical objects that are "unphysical" in that they are not in accordance with the laws or principles of physics 
according to the individual's lived experience. The human mechanism for intuitive perception is a kind of pattern detection that is trained by past patterns encountered during one's lived experience. Any such patterns can likely be detected by AI as well, which excels at pattern detection. Functional models are also semantic models. By defining a functional representation of physical systems, and by defining a semantic representation of physical theories, any physical theory can potentially be applied to any physical system by a functional model of cognition. By modeling physical systems, and by designing simulations of the behavior of those systems that reflect the current understanding of physics, any unphysical behavior encountered at any values of any parameters of the simulation might then be detectable as patterns by AI, revealing holes in current theories and enabling theories to be explored at exponentially greater levels of complexity as well as at exponentially greater speed and scale.

In the same way that objects can be represented in physical space and unphysical behavior detected, Human-Centric Functional Modeling can be used to define other functional state spaces in which "unphysical" behavior might be used to distinguish truth from falsehood. A financial state space might enable good investments to be clearly distinguished from misleading ones. In the case of medicine, every leaf or root on this earth is a potential medicine in that it might have some impact on the human system. However the incompatibility of the research methods used by different researchers, not to mention the fundamental incompatibility of the different medical traditions that might lead to researchers in one tradition failing to capture data needed to make assessments in another tradition, all combine to drag down research. Making all data that exists anywhere available to all research everywhere requires solving a number of problems such as making data sharing self-sustaining, and defining a universal data model. The state space for the human system that is currently being elaborated might provide this common model to radically increase the rate of medical discovery..

This paper has explored how HCFM might be used to define functional models and simulations of the behavior of those models reflecting all current research, that an Artificial General Intelligence (AGI) might use to exponentially increase impact on individual research outcomes, and that a General Collective Intelligence (GCI) might use to exponentially increase impact on collective research outcomes.

Increasing capacity for individual discovery can potentially be achieved through an intelligent agent acting on behalf of the individual that implementis some subset of functionality required for Artificial General Intelligence (AGI) [7]. Such an AGI might be reliably implementable with GCI [12]. Increasing capacity for collective discovery can potentially be achieved by implementing some subset of functionality required for a General Collective Intelligence [3]. Both AGI and GCI require common models for representing concepts and reasoning. These models do not need to be complex to facilitate operation even in the most complex domains such as physics or mathematics [8]. Instead their key requirement is that they be human-centric and therefore intuitive, which in turn requires that the meaning in all such models must be fully self-contained in not requiring any additional information to interpret (i.e. they must be complete semantic representations) so that reasoning processes can be decoupled in a way that enables them to be scaled over any number of functional components in an AGI, and over any number of participants in a GCI.

In addition, in order to exponentially increase capacity for discovery, more is required than having a GCI to exponentially increase ability to navigate complexity, to exponentially the number of reasoning paths the system has the capacity to navigate, as well as to exponentially increase the rate at which value can be created and the volume of value that can be created in order to sustain that navigation process. There must also be processes of discovery to apply that exponentially greater intelligence to. Such processes are required in order to methodically expand the accessible region of conceptual space. 
One general process of discovery is to generalize research problems until solutions in other domains can be found and reused. Another general process of discovery is to generalize theories uncovered by research until problems they apply to can be found in other domains where those solutions apply. A potentially unlimited number of such processes are possible. In order to target these general processes at specific research or design problems, these processes need to be modeled and decomposed into activities and roles that can be orchestrated collectively by a GCI.

\section{Implications}

With the increasing volume and complexity of information and theories in the various research disciplines, and with the increasing capacity of communication networks, the rate at which information can be transmitted is increasingly not a limit to the problem-solving ability of individuals or groups. Instead, a key limit to problem-solving ability is the rate at which understanding (as opposed to information) can be transmitted. Completely self-contained (semantic) representations of information create the potential to exponentially increase the rate at which understanding might be transmitted. The conceptual space of the Functional Modeling Framework is believed to be the first to define all of the functions required for all reasoning to be represented, and therefore has the potential to provide the first complete semantic representation as required to remove the limits to the transmission rate of understanding.

If HCFM can be used for all systems to create simple functional models that span all possible system behavior in each domain, such models might create the capacity to navigate all knowledge of such systems in this existence. By enabling AGI and GCI to exponentially increase capacity to detect which theories or components of theories provide reasoning paths that are most fit in defining problems or providing solutions in each domain, humans might gain the ability to navigate towards an exponentially larger knowledge of this existence than is currently possible. Just as computer technology executing arithmetic functions removed the burden of remembering times tables as a limit to human ability to effectively navigate the number space, cognitive computing technology executing operations in functional models has the potential to change the way humans solve problems.

Improving education, improving ability to find and support exceptional talent, might not have the capacity to reliably achieve an exponential increase in research discovery. Reliably achieving such an increase likely requires exponentially increasing capacity to consider all options. In fact, any sufficiently exceptional talent might even be limited in their capacity for discovery precisely because they are exceptional. For example, if all the knowledge of a civilization a thousand years more advanced than the current human civilization was suddenly discovered or made available, the rate at which that knowledge could be understood, published, supported by current research funding systems that often rely on publication, and then diffused by the education system, might make the required investment of time and other resources sufficiently risky that such knowledge simply could not be collectively digested.

However, implementing a GCI, and implementing an AGI (which might require GCI as well), is not a simple project that can reliably be accomplished in a top-down way. Implementing GCI appears to require deploying a self-assembling process by which engagement by increasing number of people and engagement of increasing resources can be scaled to whatever degree required [13].

\section{Conclusions}

An exponential increase in capacity for discovery might be reliably achievable with GCI, and with the AGI that might be reliably implementable with GCI. Being such a significant departure, GCI involves 
significant risks. However, an exponential increase in ability to tackle the potentially even more collective risks faced by civilizations, might justify the exploration of this area.

\section{References}

[1] Williams, A. E. (2020, May 15). Use of Human-Centric Functional Modeling to Maximize Convergence in Integrative Research. https://doi.org/10.31730/osf.io/jv6h8

[2] Williams, A. E. (2020, April 16). A Human-Centric Functional modelling Framework for Defining and Comparing Models of Consciousness and Cognition. https://doi.org/10.31234/osf.io/94gw3 [3] Williams, A. E. (2020, December 2). General Collective Intelligence vs the Innate Collective Intelligence Factor. https://doi.org/10.31730/osf.io/kp3x8

[4] Woolley, Anita Williams; Chabris, Christopher F.; Pentland, Alex; Hashmi, Nada; Malone, Thomas W. (29 October 2010). "Evidence for a Collective Intelligence Factor in the Performance of Human Groups". Science. 330 (6004): 686-688. Bibcode:2010Sci...330..686W. doi:10.1126/science.1193147. PMID 20929725. S2CID 74579.

[5] Krafft, P.M. (2019), A Simple Computational Theory of General Collective Intelligence. Top Cogn Sci, 11: 374-392.

[6] Malone, T. W. (2018). Superminds: The surprising power of people and computers thinking together.

[7] Williams A.E. (2020) A Model for Artificial General Intelligence. In: Goertzel B., Panov A., Potapov A., Yampolskiy R. (eds) Artificial General Intelligence. AGI 2020. Lecture Notes in Computer Science, vol 12177. Springer, Cham. https://doi.org/10.1007/978-3-030-52152-3_38

[8] Andy E. Williams, Detecting Truth in Conceptual Space with Artificial General Intelligence and General Collective Intelligence, working paper (2020)

[9] Andy E. Williams, Defining Semantic and Functional Models of Physical Systems to Facilitate the Use of Artificial General Intelligence and General Collective Intelligence in Physics, working paper (2020)

[10] Williams, A. E. (2020, April 27). Defining Functional Models of Artificial Intelligence Solutions to Create a Library that an Artificial General Intelligence can use to Increase General Problem Solving Ability. https://doi.org/10.31730/osf.io/hpzb7

[11] Williams, A. E. (2020, August 19). Defining Functional Models of Collective Intelligence Solutions to Create a Library a General Collective Intelligence can use to Increase General Problem Solving Ability. https://doi.org/10.31730/osf.io/q75rv

[12] Williams, A. E. (2020, August 17). Does Creating an Artificial General Intelligence Require General Collective Intelligence in Order to be Reliably Achievable?. https://doi.org/10.31730/osf.io/3q72c

[13] Williams, A. E. (2020, December 16). Discovering and Implementing Self-Sustaining Networks of Cooperation with General Collective Intelligence. https://doi.org/10.31730/osf.io/safxk 